\title{
Magnetized plasma sheath in the presence of negative ions
}

Cite as: Phys. Plasmas 27, 063520 (2020); https://doi.org/10.1063/5.0004796

Submitted: 15 February 2020 . Accepted: 02 June 2020 . Published Online: 23 June 2020

(D) R. Paul, (iD S. Adhikari, (D) R. Moulick, (D) S. S. Kausik, and B. K. Saikia
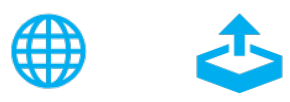

View Online

\section{ARTICLES YOU MAY BE INTERESTED IN}

Sheath formation in collisional, low pressure, and magnetized plasma

Physics of Plasmas 26, 043512 (2019); https://doi.org/10.1063/1.5090537

Influence of ionization on sheath structure in electropositive warm plasma carrying two-

temperature electrons with non-extensive distribution

Physics of Plasmas 27, 063515 (2020); https://doi.org/10.1063/5.0003242

Role of sheared $\mathrm{E} \times \mathrm{B}$ flow in self-organized, improved confinement states in magnetized plasmas

Physics of Plasmas 27, 060501 (2020); https://doi.org/10.1063/1.5142734

\section{AIP Advances Fluids and Plasmas Collection}




\title{
Magnetized plasma sheath in the presence of negative ions
}

\author{
Cite as: Phys. Plasmas 27, 063520 (2020); doi: 10.1063/5.0004796 \\ Submitted: 15 February 2020 - Accepted: 2 June 2020 . \\ Published Online: 23 June 2020
}

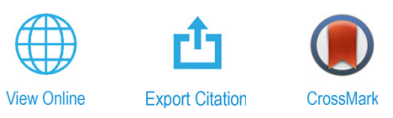

\author{
R. Paul, , (D) S. Adhikari, ${ }^{1,2}$ (D) R. Moulick, ${ }^{3}$ (D) S. S. Kausik, ${ }^{1, a)}$ (D) and B. K. Saikia
}

\author{
AFFILIATIONS \\ ${ }^{7}$ Centre of Plasma Physics, Institute for Plasma Research, Sonapur 782402, Kamrup (M), Assam, India \\ ${ }^{2}$ Department of Physics, University of Oslo, PO Box 1048 Blindern, NO-0316 Oslo, Norway \\ ${ }^{3}$ Lovely Professional University, Jalandhar Delhi-GT Road, Phagwara 144411, Punjab, India \\ ${ }^{a)}$ Author to whom correspondence should be addressed: kausikss@rediffmail.com
}

\begin{abstract}
The sheath formation in a weakly magnetized collisionless electronegative plasma consisting of electrons and negative and positive ions has been numerically investigated using the hydrodynamic equations. The electrons and negative ions are assumed to follow the Boltzmann relation. A sheath formation criterion has been analytically derived. This paper focuses on studying the sheath structure by varying the electronegativity. It has been observed that the presence of negative ions has a substantial effect on the sheath structure. The observations made in the present work have profound significance on processing plasmas, specifically in the semiconductor industry as well as in fusion studies.
\end{abstract}

Published under license by AIP Publishing. https://doi.org/10.1063/5.0004796

\section{INTRODUCTION}

The physics of sheaths has been intriguing to researchers for several decades as it harbors diverse physical phenomena. The sheath structure, both in an electrostatic scenario and in the magnetized case, is widely studied. ${ }^{1}$ The knowledge of sheaths helps in understanding the interaction of plasmas with different material surfaces. ${ }^{2}$ Besides being one of the oldest topics in plasma, it finds its application in plasma technology, fusion research, and many more. ${ }^{3}$ In fusion devices such as tokamaks, the magnetic field often makes an angle with the boundary wall. With the ongoing developments in fusion research, the study of the sheath in the oblique or tilted magnetic field has gained utmost importance. . $^{1,4-6}$

It is well known that in the absence of the magnetic field, a nonneutral region appears near the wall. It consists of two subregions, namely, the presheath and the sheath region. The presheath is located near the bulk plasma, whereas the sheath lies closer to the wall. In the absence of sources, the positive ion flux remains constant in the sheath. The sheath so formed is known as the Debye sheath. Usually, the sheath begins when the ions satisfy the Bohm criterion given by

$$
v_{i} \geq c_{s}=\sqrt{\frac{T_{e}}{m_{i}}},
$$

where $v_{i}$ and $c_{s}$ are the positive ion velocity at the sheath edge and the Bohm speed, and $T_{e}$ and $m_{i}$ are the electron temperature and positive ion mass, respectively. On the other hand, in the presence of the magnetic field, adjacent to the Debye sheath, another layer forms known as the magnetic presheath or Chodura sheath. If plasma contains negative ions as one of its components, then the sheath structure has been observed to be modified, ${ }^{7,8}$ and such plasmas are termed as electronegative plasmas. For such plasma, the sheath potential was studied by Thompson and Boyd in 1950s.

Electronegative gases and plasmas have been seen to gather a lot of attention in recent times as they have applications in different fields, such as surface processing, atmospheric science, environmental studies, and many others. ${ }^{10,11}$ These electronegative plasmas are also used as negative ion beam generators, which are used as neutral beams in fusion reactors. ${ }^{10,12}$ The presence of the negative ions is also said to improve the performance of dry etching, ${ }^{13}$ which enables producing integrated circuits in bulk. ${ }^{14}$ In low-pressure plasmas, the negative ions are usually created by volume production, ${ }^{15,16}$ but the negative ions are also produced by surface production as well. ${ }^{12}$ Recently, Kakati et al. ${ }^{17}$ have developed a novel process of producing negative hydrogen ions $\left(H^{-}\right)$by cesium coated tungsten dust. They have reported of producing the $H^{-}$ions by the surface process as it has an advantage over the two-step volume process. ${ }^{17}$

One of the important parameters for defining negative ion plasmas is electronegativity $\left(\alpha=\frac{n_{-}}{n_{e}}\right)$. Many researchers have studied electronegative plasmas in the presence of an external magnetic field. 
Yasserian and Aslaninejad ${ }^{14}$ studied the presheath-sheath in magnetized electronegative discharge in the presence of magnetic field and elastic collisions including ion-neutral collisions. Hatami et al. ${ }^{8}$ studied the sheath in the presence of negative ions in a multicomponent plasma considering two species of positive ions and electrons neglecting the effect of collisions, ionization, and recombination. Franklin and Snell, ${ }^{18}$ on the other hand, studied the sheath structure in the presence of an external magnetic field but with only one species of positive and negative ions each. Besides the fluid approach, the kinetic approach has also been considered in studying the electronegative plasmas. ${ }^{19}$

While studying the sheath structure for electronegative plasmas, stratified structures have been observed by many researchers. ${ }^{20-22}$ The stratification of electronegative plasmas was initially studied by Lampe et al., ${ }^{23}$ and their work implies that addition of an infinitesimal electronegative gas to otherwise electropositive plasma gives rise to a strongly electronegative core. Moreover, there is an abrupt spatial transition from the more electronegative core to a weakly electronegative core. In the model equations, they considered the standard continuity and momentum equations for the negative ions in the presence of an electrostatic term alone. A similar investigation has been reported by Moulick and Goswami ${ }^{24}$ even in the presence of collisions. They have concluded that the negative ions are mostly bounded in the bulk region and hardly travel toward the sheath. Edgley et $a .^{25}$ and Deutsch and Räuchle ${ }^{26}$ also have drawn similar conclusions. All of these studies, however, do not consider the magnetic field and have found continuous evolution of the electric potential. The region of simulation in all of these studies is similar, which means the numerical integration of the governing equations is started at the bulk plasma and is carried forward to the wall.

On the other hand, Yasserian et al..$^{14,27,28}$ have investigated the sheath region of electronegative plasma and found the double layered stratified structure. The model is quite similar to the present one. The magnetic field lies in the $\mathrm{x}-\mathrm{z}$ plane with the electric field along the $\mathrm{z}$ direction. Thus, the sheath forms along the z-direction. However, it is to be noted that the initial ion velocities along $\mathrm{x}$ - and $\mathrm{y}$-direction are put to zero, while starting the numerical integration. But these two components of the ion velocities do share a significant portion of the total ion velocity. This, in turn, changes the overall ion dynamics in the presence of a magnetic field. The resulting velocity sharing and sheath formation has been discussed in detail by Moulick et al. ${ }^{1,29}$ In order to get the appropriate initial conditions for all the components of ion velocity, the Taylor series expansion must be done at the midplane of the plasma, and the sum of the first few terms should be considered. It is not only important for the magnetized plasma but also for the electrostatic plasma (most importantly if the solution is sought from the midpoint or bulk of the plasma). ${ }^{25,30,31}$ Otherwise, the solutions may deviate from being continuous and monotonic.

In addition to the above-mentioned studies, the effect of negative ion temperature and electronegativity seems to have a notable influence on the sheath structure. In the present work, a collionless, lowtemperature electronegative plasma has been investigated using the fluid approach. The current work focuses on studying the effect of varied electronegativity on the sheath structure. It also emphasizes the impact of the negative-ion temperature in understanding the dynamics involved in the formation of the sheath. One of the novelties of this study is the derivation of the generic sheath formation criterion for an electronegative plasma in the presence of magnetic field.

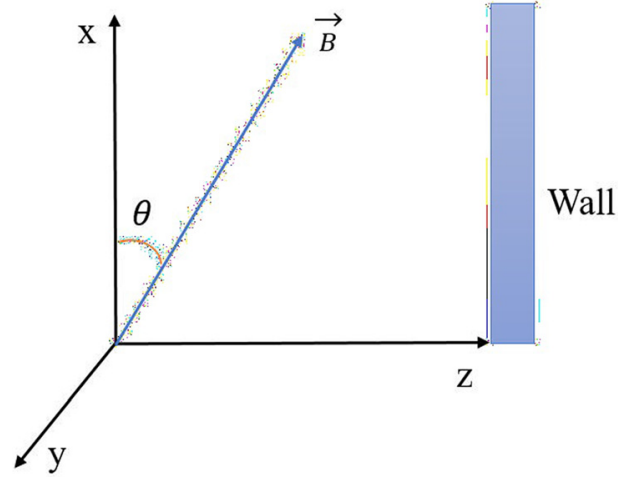

FIG. 1. Geometry of the system.

This paper has been divided into the following sections: Sec. II discusses the model and the set of equations used in modeling the system. Sections III and IV analyze the numerical findings, and a brief conclusion has been presented in Sec. V.

\section{MODEL AND BASIC EQUATIONS}

A multi-component magnetized plasma has been considered comprised of electrons, positive ions, and negative ions. The plasma is considered to be in contact with a planar wall, as shown in Fig. 1. An oblique magnetic field $\mathbf{B}$ considered lies in the $\mathrm{x}-\mathrm{z}$ plane with an angle of inclination, $\theta$ to the $\mathrm{x}$-axis perpendicular to the wall. The surface is parallel to the $\mathrm{x}$-axis. The plasma is assumed to be in steady-state, and the ion-neutral collisions have been neglected.

The electrons and negative ions can be described by the Boltzmann relation, which is given by

$$
n_{e}=n_{e o} \exp \frac{e \phi}{k T_{e}},
$$
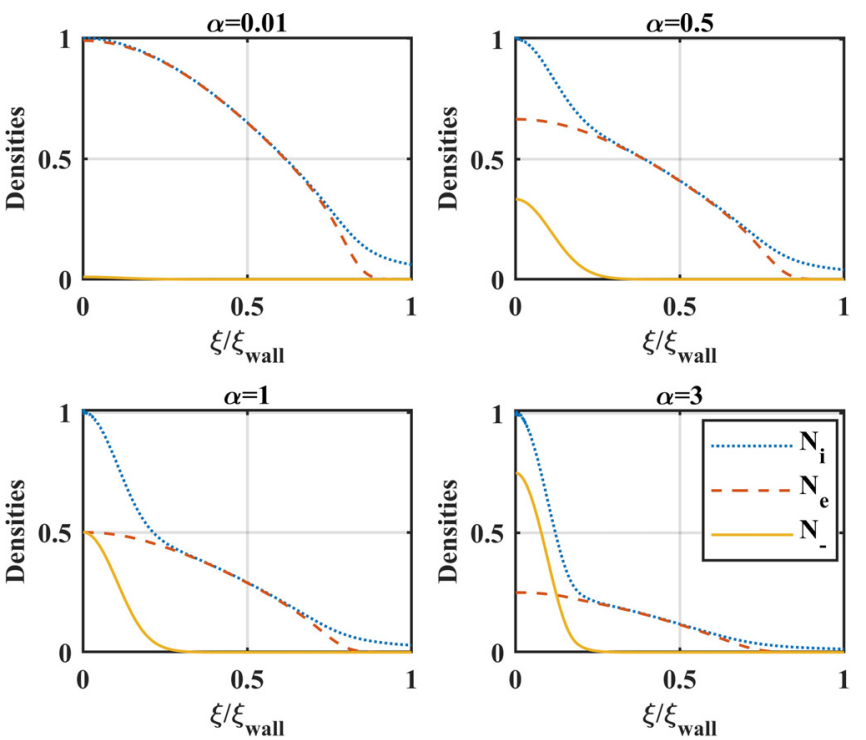

FIG. 2. Normalized density profile of all the species along the normalized $z$ axis. 


$$
n_{-}=n_{-o} \exp \frac{e \phi}{k T_{-}},
$$

where $T_{e}$ and $T_{-}$represent the temperature of the electrons and the negative ions, respectively, $\phi$ is the electric potential, and $n_{e o}$ and $n_{-o}$ represent the density of the electrons and negative ions, respectively. An isotropic medium has been considered, where all species have uniform temperature in all directions. The anisotropic ion temperature is often observed in magnetized plasma. The anisotropic heating of the ions has long been understood to be a result of collisionless heating of the ions by turbulence, resulting from numerous instabilities within the plasma. In addition, the resonant interaction of ions with ion cyclotron waves is another strong mechanism for such anisotropic heating. Moreover, the anisotropy in ion temperature is found in space plasmas, specifically in the high altitude F-region of the ionosphere. ${ }^{32,33}$ The anisotropy has a significant role to play and acts like an agent for exciting numerous instabilities in the plasma. In contrast, the plasma considered in this paper is considered to be devoid of any such instability as it is thought to be typical of processing plasmas. The prime objective is to observe the ion distributions near the wall and to get a generic criterion for the sheath formation for the electronegative plasma in the presence of magnetic field. The ion temperature is, therefore, taken to be significantly lower than the electron temperature, and any existing anisotropy is neglected.

The electrons are chosen to be Boltzmannian due to their negligible mass. Apart from this approach, the electrons being described by Boltzmann relation can be justified when the pressure gradient balances the electric force. ${ }^{34}$ This relation is a consequence of the absence of the magnetic field, collision, and particle generation in the momentum balance equation for electrons. In the absence of the other factors, the presence of the magnetic field alone may be sufficient to cause the deviation from the Boltzmann behavior. ${ }^{35}$ However, in case the electrons are strongly magnetized, the fluid velocity is the electrons is small compared to the thermal velocity, and this retains the Boltzmann behavior. According to Chen, ${ }^{36}$ the Maxwellian distribution can be considered in the presence of a dc magnetic field as the Lorentz force is perpendicular to the velocity; thus, it is unable to impart energy to any electron.

Negative ions as well have been described by the Boltzmann relation. The electrons and the negative ions are pushed by the electric field back toward the center of the discharge in the presheath region, which decreases the velocities of the electrons and the negative ions. The magnetic field force has a negligible effect on the negative ions as compared to the impact on the positive ions. ${ }^{7}$ This aids in the balance between the drift and the diffusion, thereby validating the description of negative ions by the Boltzmann relation. by

The cold ions are governed by the hydrodynamic equations given

$$
m_{i} n_{i}(\mathbf{v} . \nabla) \mathbf{v}=n_{i} e(\mathbf{E}+\mathbf{v} \times \mathbf{B})-m_{i} S_{i} \mathbf{v} .
$$

The continuity equation is given by

$$
\nabla \cdot\left(n_{i} \mathbf{v}\right)=S_{i},
$$

where

$$
S_{i}=n_{e} \mathbb{Z}
$$

Here, $\mathbf{B}$ is the magnetic field intensity, $\mathbb{Z}$ is the ionization rate which is assumed to be constant, and $m_{i}$ and $v$ are the mass and velocity of the positive ions, respectively.

Considering the $1 \mathrm{D}-3 \mathrm{~V}$ representation of the system, the equations can be resolved into components along the $x^{-}, y_{-}^{-}$, and $z_{-}^{-}$ directions as

$$
\begin{gathered}
v_{z} \frac{d v_{x}}{d z}=\omega_{z} v_{y}-\frac{n_{e} \mathbb{Z}}{n_{i}} v_{x}, \\
v_{z} \frac{d v_{y}}{d z}=\left(\omega_{x} v_{z}-\omega_{z} v_{x}\right)-\frac{n_{e} \mathbb{Z}}{n_{i}} v_{y}, \\
v_{z} \frac{d v_{z}}{d z}=-\frac{e}{m_{i}} \frac{d \phi}{d z}-\omega_{x} v_{y}-\frac{n_{e} \mathbb{Z}}{n_{i}} v_{z}, \\
\frac{d\left(n_{i} v_{z}\right)}{d z}=n_{e} \mathbb{Z} .
\end{gathered}
$$

The electrostatic potential is governed by Poisson's equation

$$
\frac{d^{2} \phi}{d z^{2}}=-\frac{e}{\varepsilon_{o}}\left(n_{i}-n_{e}-n_{-}\right) .
$$

To solve the above set of equations, it is necessary to normalize the quantities by the following dimensionless parameters:

$$
\begin{gathered}
u=\frac{v_{x}}{c_{s}}, \quad v=\frac{v_{y}}{c_{s}}, \quad w=\frac{v_{z}}{c_{s}}, \\
\gamma_{x}=\frac{\omega_{x} \lambda_{n i}}{c_{s}}, \quad \gamma_{z}=\frac{\omega_{z} \lambda_{n i}}{c_{s}}, \quad \beta=\frac{\mathbb{Z} \lambda_{n i}}{c_{s}}, \\
\xi=\frac{z}{\lambda_{n i}}, \quad \eta=-\frac{e \phi}{k T_{e}}, \quad \lambda_{n i}=\frac{c_{s}}{\mathbb{Z}}, \\
N_{i}=\frac{n_{i}}{n_{i o}}, \quad N_{e}=\frac{n_{e}}{n_{i o}}, \quad N_{-}=\frac{n_{-}}{n_{i o}}, \quad \alpha=\frac{n_{-o}}{n_{e o}}, \\
\delta=\frac{n_{e o}}{n_{i o}}, \quad 1-\delta=\frac{n_{-o}}{n_{i o}}, \tau_{-}=\frac{T_{e}}{T_{-}},
\end{gathered}
$$

where

$$
\begin{gathered}
c_{s}=\sqrt{\frac{k T_{e}}{m_{i}}}, \quad \lambda_{D}=\sqrt{\frac{\varepsilon k T_{e}}{n_{e o} e^{2}}}, \\
\omega_{x}=\frac{e B_{x}}{m_{i}}=\frac{e B_{o} \cos \theta}{m_{i}}, \\
\omega_{z}=\frac{e B_{z}}{m_{i}}=\frac{e B_{o} \sin \theta}{m_{i}},
\end{gathered}
$$

where $\omega_{x}$ and $\omega_{z}$ are the respective ion-cyclotron frequencies along the $\mathrm{x}$ - and $\mathrm{z}$-directions.

Here, the equations are scaled in the ionization scale as the presheath region is also considered.

The normalized equations are given by

$$
\begin{gathered}
\frac{d u}{d \xi}=\gamma_{z}\left(\frac{v}{w}\right)-\beta \frac{N_{e}}{N_{i}} \frac{u}{w}, \\
\frac{d v}{d \xi}=\gamma_{x}-\gamma_{z}\left(\frac{u}{w}\right)-\beta \frac{N_{e}}{N_{i}} \frac{v}{w}, \\
\frac{d w}{d \xi}=\frac{1}{w} \frac{d \eta}{d \xi}-\gamma_{x}\left(\frac{v}{w}\right)-\beta \frac{N_{e}}{N_{i}}, \\
\frac{d N_{i}}{d \xi}=2 \beta \frac{N_{e}}{w}-\frac{N_{i}}{w^{2}} \frac{d \eta}{d \xi}-\gamma_{x}\left(\frac{N_{i}}{w^{2}}\right) v,
\end{gathered}
$$




$$
\frac{d^{2} \eta}{d \xi^{2}}=a_{o}\left(N_{i}-\delta N_{e}-(1-\delta) N_{-}\right)
$$

where

$$
a_{o}=\left(\frac{\lambda_{n i}}{\lambda_{D}}\right)^{2} \text {. }
$$

The normalized electron and negative ion densities are given by

$$
N_{e}=\exp (-\eta), \quad N_{-}=\exp \left(-\eta \tau_{-}\right) .
$$

\section{A. The sheath formation criterion}

Usually for a collisionless unmagnetized plasma, the sheath is formed when the ions are sonic at the edge, which is termed as the Bohm criterion for the sheath formation. In the presence of the magnetic field, however, it is observed that the condition is modified. The sheath formation criterion for the present model has been analytically derived. The space charge variation is often defined as

$$
\sigma=n_{i}-n_{e}-n_{n}
$$

and variation along the $z$-direction is given by

$$
\begin{gathered}
\frac{d \sigma}{d z}=\frac{d\left(n_{i}-n_{e}-n_{-}\right)}{d z} \text { or, } \\
\frac{d \sigma}{d z}=\frac{n_{i}}{v_{z}}\left[\Omega-\frac{e E}{m_{i} v_{z}}+\frac{v_{y}}{v_{z}} \omega_{x}\right]+\frac{n_{e} e E}{T_{e}}+\frac{n_{-} e E}{T_{e}} \tau_{-},
\end{gathered}
$$

where

$$
\Omega=\frac{2 n_{e} \mathbb{Z}}{n_{i}}
$$

For sheath formation,

$$
\begin{gathered}
\frac{d \sigma}{d z}>0 \\
\text { or, } \frac{n_{i}}{v_{z}}\left[\Omega-\frac{e E}{m_{i} v_{z}}+\frac{v_{y}}{v_{z}} \omega_{x}\right]+\frac{n_{e} e E}{T_{e}}+\frac{n_{-} e E}{T_{e}} \tau_{-}>0, \\
\text { or, }\left[1-v_{z}^{2}\left(\frac{1}{v_{c}^{2}}+\frac{\tau_{-}}{v_{n}^{2}}\right)\right]<\frac{v_{z}}{v_{\Omega}}\left[1+\frac{v_{y}}{v_{z}} \frac{\omega_{x}}{\Omega}\right]
\end{gathered}
$$

where

$$
v_{\Omega}=\frac{e E}{m_{i} \Omega}, \quad v_{c}=v_{B} \sqrt{\frac{n_{i}}{n_{e}}}, \quad v_{n}=v_{B} \sqrt{\frac{n_{i}}{n_{-}}} .
$$

Equation (17) represents the general condition for the sheath formation. It is seen that the sheath criterion depends on the ion-cyclotron frequency, the respective densities of positive ions, electrons, and negative ions, and also on the temperature ratio between the electrons and the negative ions.

The wall is assumed to be floating; thus, the flux balance has been given by

$$
n_{i} v_{z}=\frac{1}{4} n_{e} \bar{c},
$$

where $\bar{c}=\sqrt{\frac{8 T_{e}}{\pi m_{e}}}$, which is the random velocity of the electron at the wall. This flux balance condition is acquired by the equating the ion drift and the random thermal motion of the electrons. ${ }^{1}$

\section{NUMERICAL EXECUTION}

Equations (10)-(14) are a set of ordinary differential equations which can be solved using the Runge-Kutta fourth order method. These are initial valued problem and the values of the dependent variable must be supplied at the midplane (initial point) of the plasma. Considering $\xi=0$ as the initial point, the quantities such as velocities and densities become zero. Hence, a point close to $\xi=0$ is considered where the initial values are obtained using Taylor series expansion. ${ }^{1,30,37}$

The series may be constructed as

$$
\begin{aligned}
V_{i} & =V_{i 1} \xi+V_{i 2} \xi^{3}+\cdots, \\
\eta & =\eta_{1} \xi^{2}+\eta_{2} \xi^{4}+\cdots,
\end{aligned}
$$

where $\mathrm{N}, \mathrm{V}$, and $\eta$ represents the density, velocity, and potential, respectively, for ions.

The first order coefficients of the above are

$$
\begin{gathered}
\eta_{1}=0, \\
u_{1}=\frac{\gamma_{x} \gamma_{z} N_{e o}}{4 N_{e o}^{2}+\gamma_{z}^{2}}, \\
v_{1}=\frac{2 \gamma_{x} N_{e o}^{2}}{4 N_{e o}^{2}+\gamma_{z}^{2}}, \\
w_{1}=\frac{N_{e o}}{N_{i o}}, \\
N_{i 0}=1 .
\end{gathered}
$$

These coefficients become the initial values and are used to obtain the solution of the above differential equations. The initial point is considered nearby $\xi=0$, as small as possible, on the order of $10^{-4}$ or $10^{-5}$.

The default parameters chosen for simulation are

$$
\begin{gathered}
T_{e}=1 \mathrm{eV} ; \quad m_{i}=1 \mathrm{AMU} ; \quad \mathbb{Z}=1 \times 10^{5} \mathrm{~s}^{-1} ; \\
n_{o}=1 \times 10^{16} \mathrm{~m}^{-3} ; \quad B_{0}=0.35 \mathrm{~T} ; \quad \theta=20^{\circ} ; \quad \tau_{-}=25 .
\end{gathered}
$$

Here, $\mathrm{H}_{2}$ plasma is considered; the positive as well as the negative ions are that of $\mathrm{H}_{2}$.

The differential equations are, thus, solved using MATLAB routine ODE45.

\section{RESULTS AND DISCUSSIONS}

\section{A. Effect of electronegativity on sheath structure}

By varying the electronegativity, the densities of the species are studied as shown in Fig. 2, and the obtained regimes can be broadly classified into three categories as follows.

\section{Weakly electronegative}

For $\alpha=0.01$, in this case, the density profile resembles that of an electron-ion plasma. The negative ion density is negligible in this case. For $\alpha=0.5$, there is a nominal negative ion density, but the more substantial portion of the density is that of electrons and that of positive ions. The particle density in the sheath, in this case, is more than that of $\alpha=0.01$, indicating in a thicker sheath than for $\alpha=0.01$. Hence, for $\alpha<1$, the plasma is said to be electron-rich plasma. 


\section{Moderately electronegative}

For $\alpha=1$, the electrons and negative ions are seen to have equal densities. The negative ion density increases with an increase in electronegativity. The particle density also increases which, in turn, further increases the sheath width.

\section{Highly electronegative}

In this regime, $\alpha=3$, a rise in the negative ion density is observed. The major contributor to the negative species, in this case, is the negative ions. Electron density decreases significantly, but the particle density increases manifold in the sheath, thereby increasing the thickness of the sheath. Therefore, for $\alpha>1$, the plasma is said to be negative ion rich plasma.

Figure 3 represents the normalized space charge density by varying the electronegativity. The space charge profile becomes useful in understanding the sheath thickness; it is seen that for a lower value of electronegativity, the space charge is comparatively higher than that for the higher values of $\alpha$. It signifies that a thin sheath is obtained for a lower electronegativity, which gradually increases with an increase in the electronegativity. For a lower value of $\alpha$, the wall becomes more negative as the electrons reach the wall faster. It results in greater charge separation and consequently higher electric field, which, in turn, decreases the sheath width. As the electronegativity increases, this charge separation decreases, and the sheath becomes thicker. Particularly for $\alpha=3$, the sheath width is maximum. With the wall being already negative due to the mobile electrons, the positive ions are attracted toward the wall. The velocity of the positive ions increases toward the wall. In the presence of the negative ions, the electron distribution widens, and the fall becomes slower as compared to the other electronegative cases. It increases the particle density in the sheath and, hence, widens the sheath.

In Fig. 4, the sheath thickness is seen to have dependence on the angle of inclination. For a collisionless case, in a magnetized scenario, the sheath thickness is observed to be wider for a low angle rather than for a higher value. Such an observation was earlier observed by

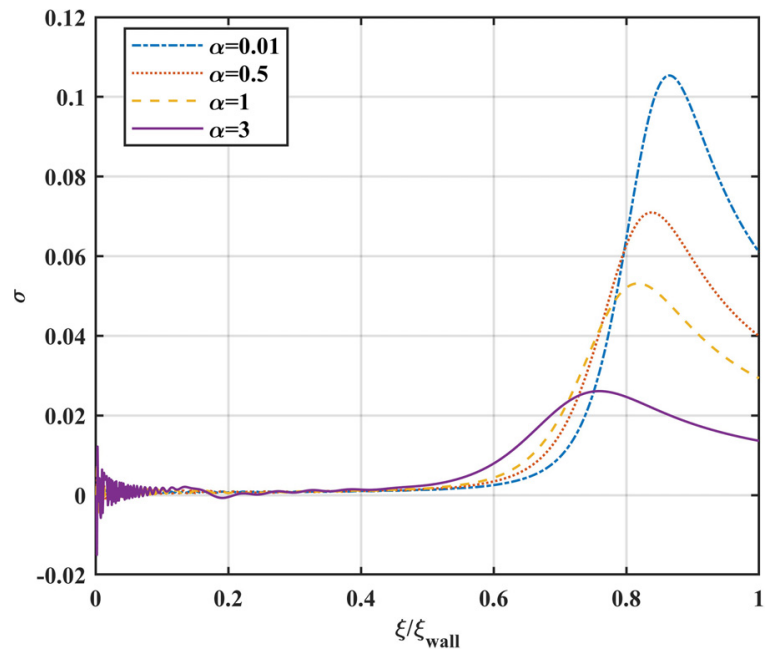

FIG. 3. Space charge variation along normalized $z$ axis for different $\alpha$ and $\theta=20^{\circ}$.

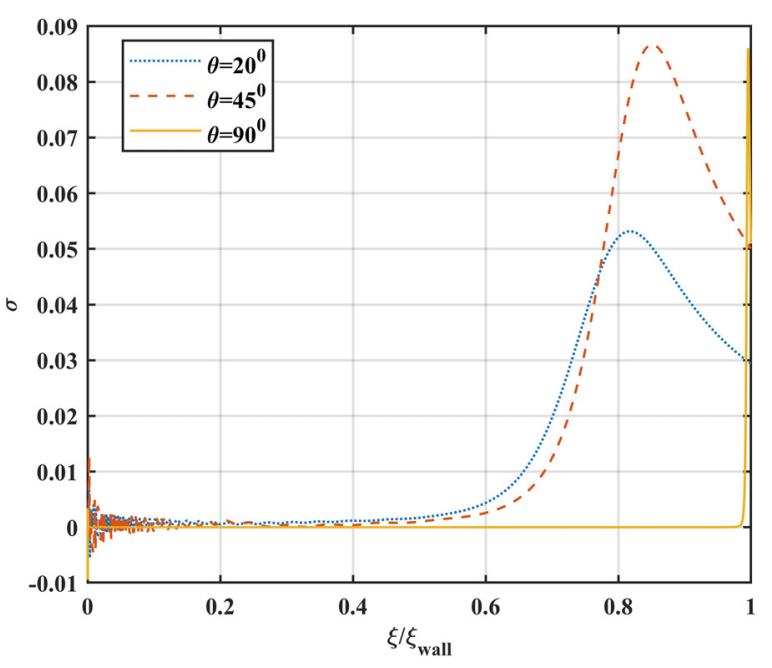

FIG. 4. Space charge variation along the normalized $z$ axis for different $\theta$ for $\alpha=1$.

Moulick et al. ${ }^{1}$ However, in the present case, it is observed that the sheath is widely expanded as compared to the electrostatic case. This is little unlike the previous observation and may be induced by the presence of the negative ions.

The space charge profile is shown in Fig. 5 for the electrostatic case by varying electronegativity. It is seen that a thin sheath is formed for lower electronegativity, which increases with an increase in $\alpha$. For electrostatic case, without collisions, the electric field force is the only possibility which affects the sheath formation. Since with the increase in $\alpha$ the electron population decreases, the probability of the electrons reaching the wall also decreases. It results in a less negative wall, consequently decreasing the electric field strength as reflected in the potential profile in Fig. 6 . Thus, the widening of the space charge region attributes to the decrease in the field strength.

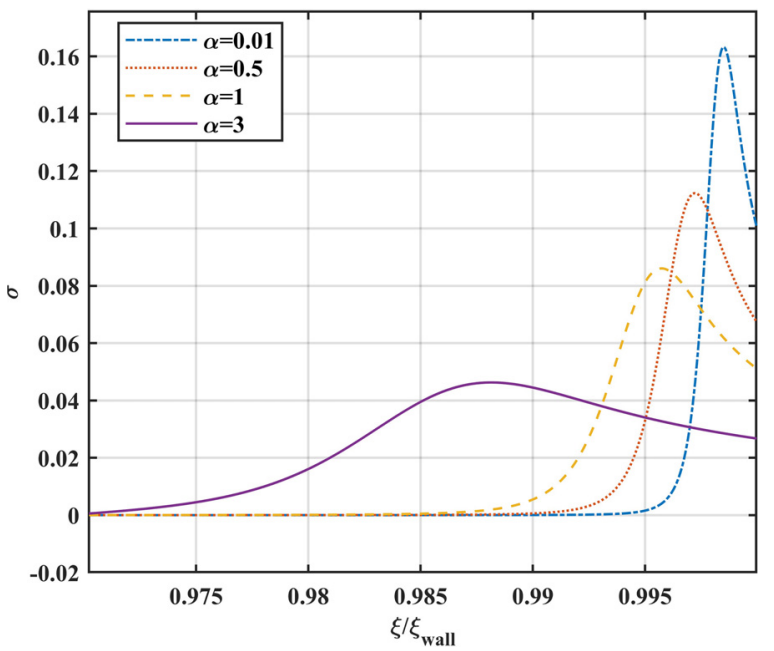

FIG. 5. Space charge variation along normalized $z$ axis for different $\alpha$ for $\theta=90^{\circ}$. 


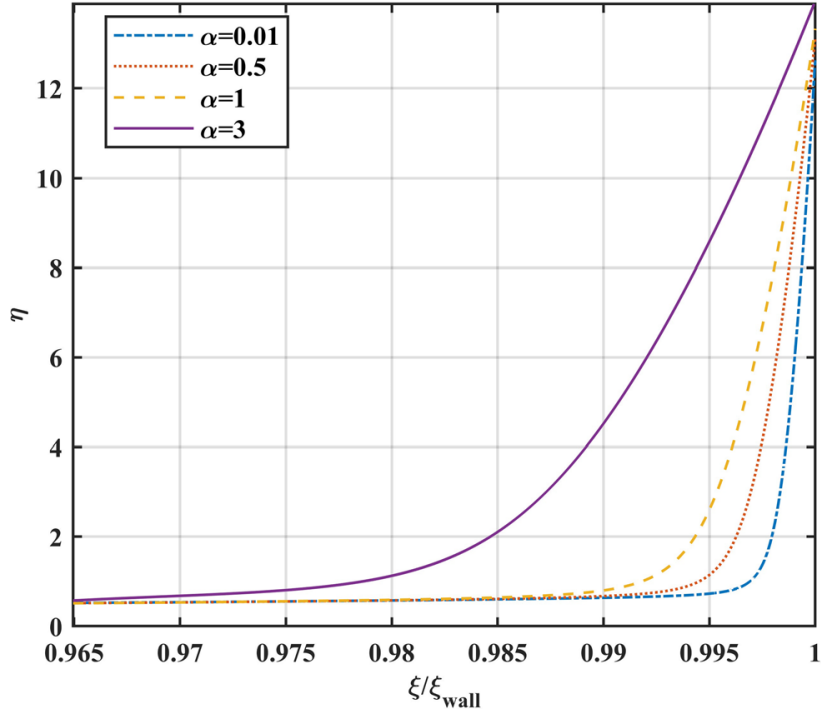

FIG. 6. Electric potential variation along normalized $z$ axis for different $\alpha$ for $\theta=90^{\circ}$.

From Fig. 2, it is seen that the negative ion density has a sharp fall inside the sheath in comparison to the electrons. It is due to the low-temperature of the negative ions. The positive ions tend to follow either the electrons or the negative ions as seen in Fig. 7. For $\alpha=0.01$, the positive ions follow the electrons. As the electronegativity increases the positive ions initially follow the negative ions, and when the negative ions fall, they tend to follow the electrons. The negative ions affect the dynamics of the positive ions.

The normalized velocity along the $z$-direction shows an irregularity for $\alpha=3$ as shown in Fig. 8. In Fig. 9, the positive ion flux also shows a deformation in the same region. The positive ion density dips in the same region as seen in Fig. 7 and its velocity along the

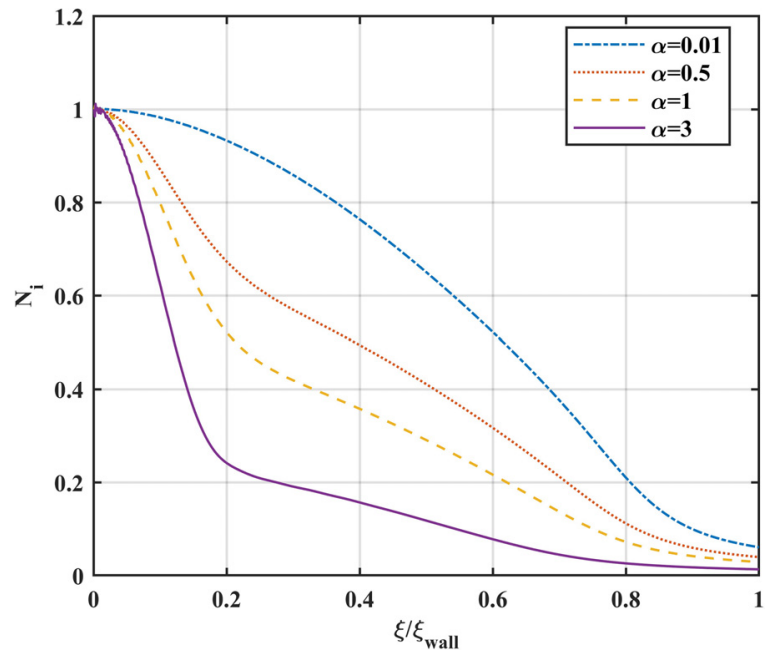

FIG. 7. Normalized positive ion density variation along the normalized $z$ axis.

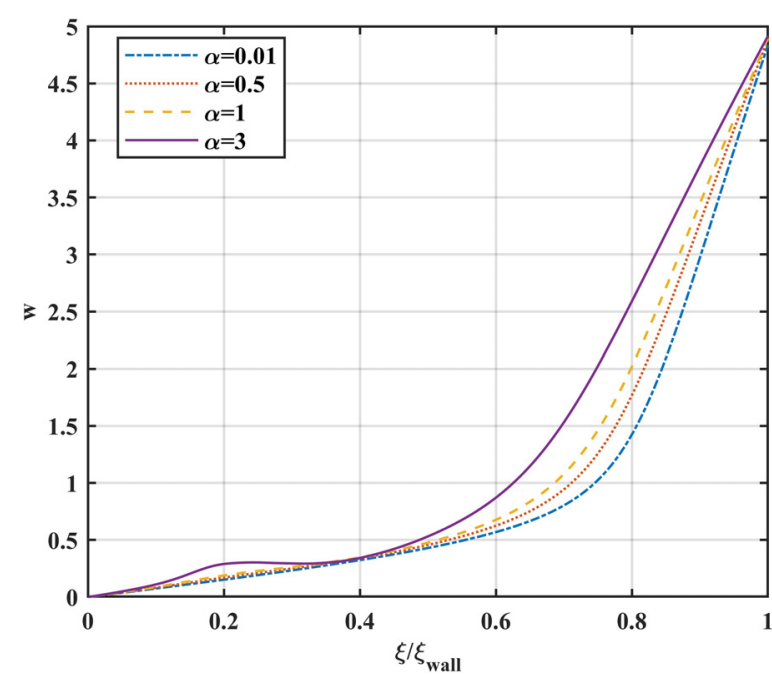

FIG. 8. Normalized positive ion velocity along the z-direction.

$z$-direction increase. Thus, the positive ion flux increases in this region. The velocity of the ions increases toward the wall which is reflected in the ion flux profile, and it becomes constant inside the sheath.

The Lorentz force is shown in Fig. 10 for different electronegativities. It is seen that for all the cases, the magnetic force along the $y$-direction increases. $F_{y}$ depends on $\gamma_{x}, w, \gamma_{z}$, and $u$. The magnitude of $\gamma_{x}$ and $w$ is much greater than that of $\gamma_{z}$ and $u$. Therefore, $F_{y}$ increases monotonically in all cases. The magnetic force helps in guiding the positive ions to enter the sheath. On reaching the sheath edge, the magnetic force no longer plays a major role. On the contrary, the electric force now accelerates the ions toward the wall. ${ }^{38}$ With the increase in electronegativity, the electric force is seen to decrease. This decrease in electric force lowers the charge separation, which further increases the particle density in the sheath and, therefore, increases the sheath width.

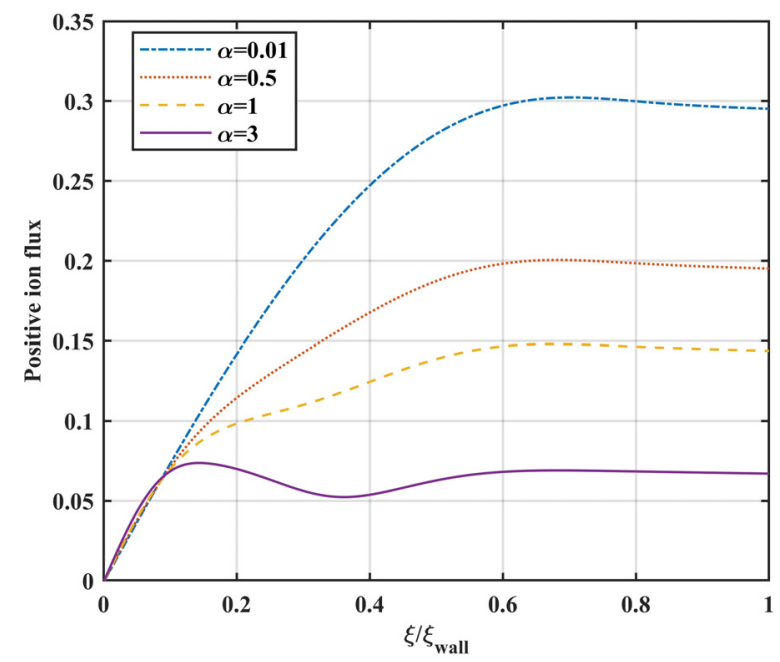

FIG. 9. Normalized positive ion flux variation along the normalized $z$ axis. 

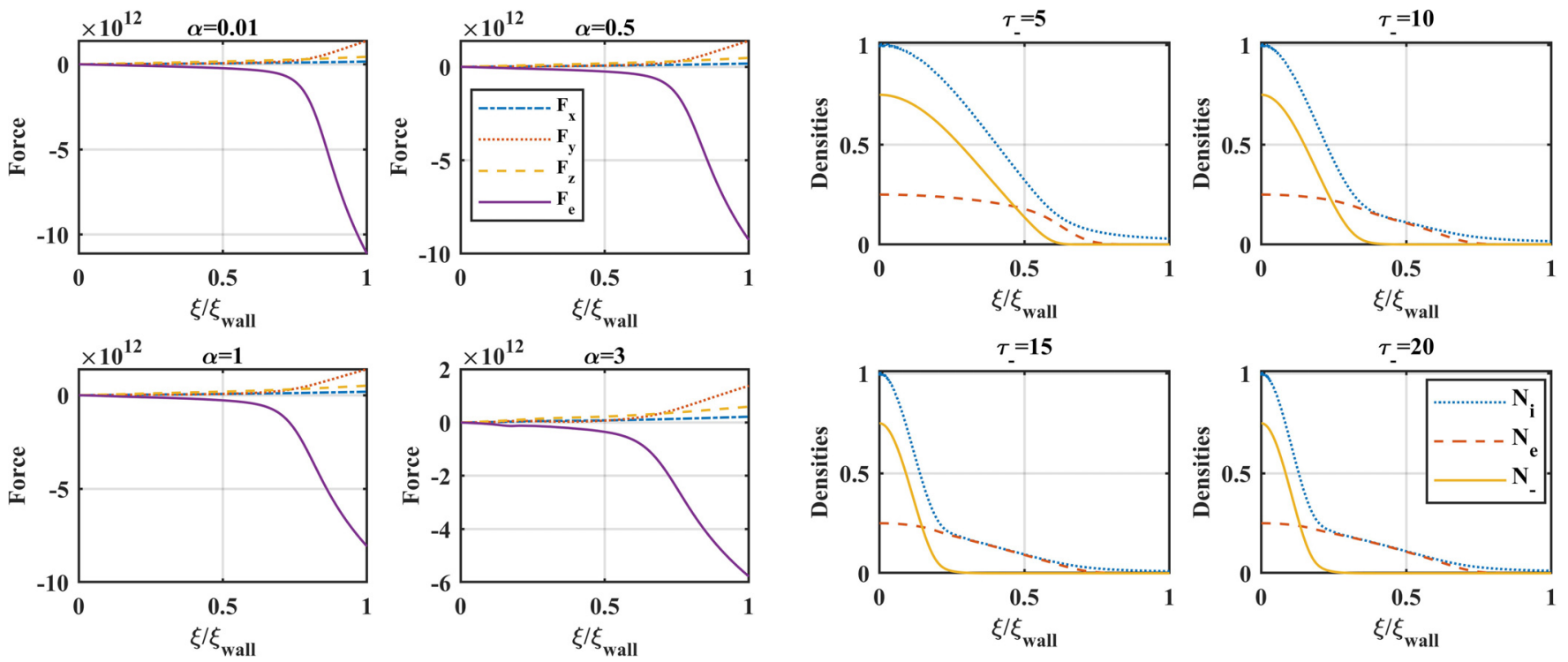

FIG. 10. Normalized Lorentz force variation along normalized $z$ axis.

For $\alpha=3$, the electric force is minimum as the charge separation is the least; hence, the sheath width is maximum in this case.

With the increase in the electronegativity, it is observed that the $y$ component of velocity increases in all the cases. From Eq. (18), it has been derived that the sheath formation criterion depends on the $y$ component of velocity. Figure 11 shows that the $y$ component of velocity increases with $\alpha$. The increase in $v_{y}$ is due to the $\mathbf{E} \times \mathbf{B}$ drift. It acts perpendicular to the $x-z$ plane, thereby increasing $v_{y}$ monotonically.
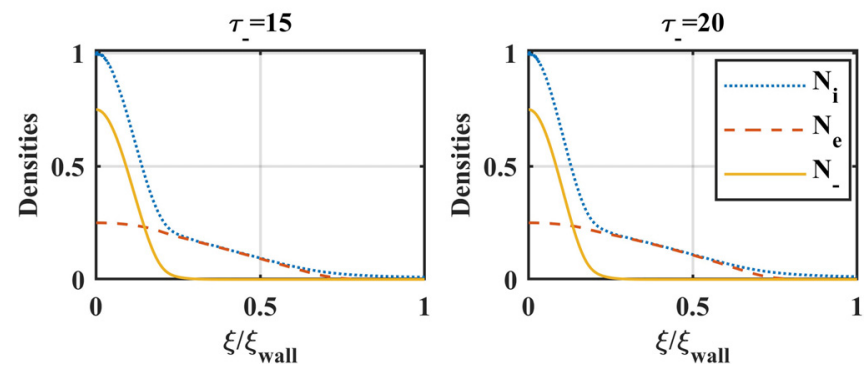

FIG. 12. Normalized species density variation along the normalized $z$ axis for different $\tau_{-}$for $\alpha=3$.

\section{B. Effect of temperature ratio on sheath structure}

For different values of $\tau_{-}$, the species density is studied as shown in Fig. 12 for $\alpha=3$. As the negative ions become colder, for increasing value of $\tau_{-}$, they tend to fall faster near the source, which facilitates the electrons toward the sheath. This increases the particle density inside the sheath and, thus, widens the sheath.

The effect of $\tau_{-}$is visible on the Lorentz force as shown in Fig. 13. On increasing $\tau_{-}$, the charge separation is seen to decrease, wherein the wall becomes less negative, thereby reducing the electric
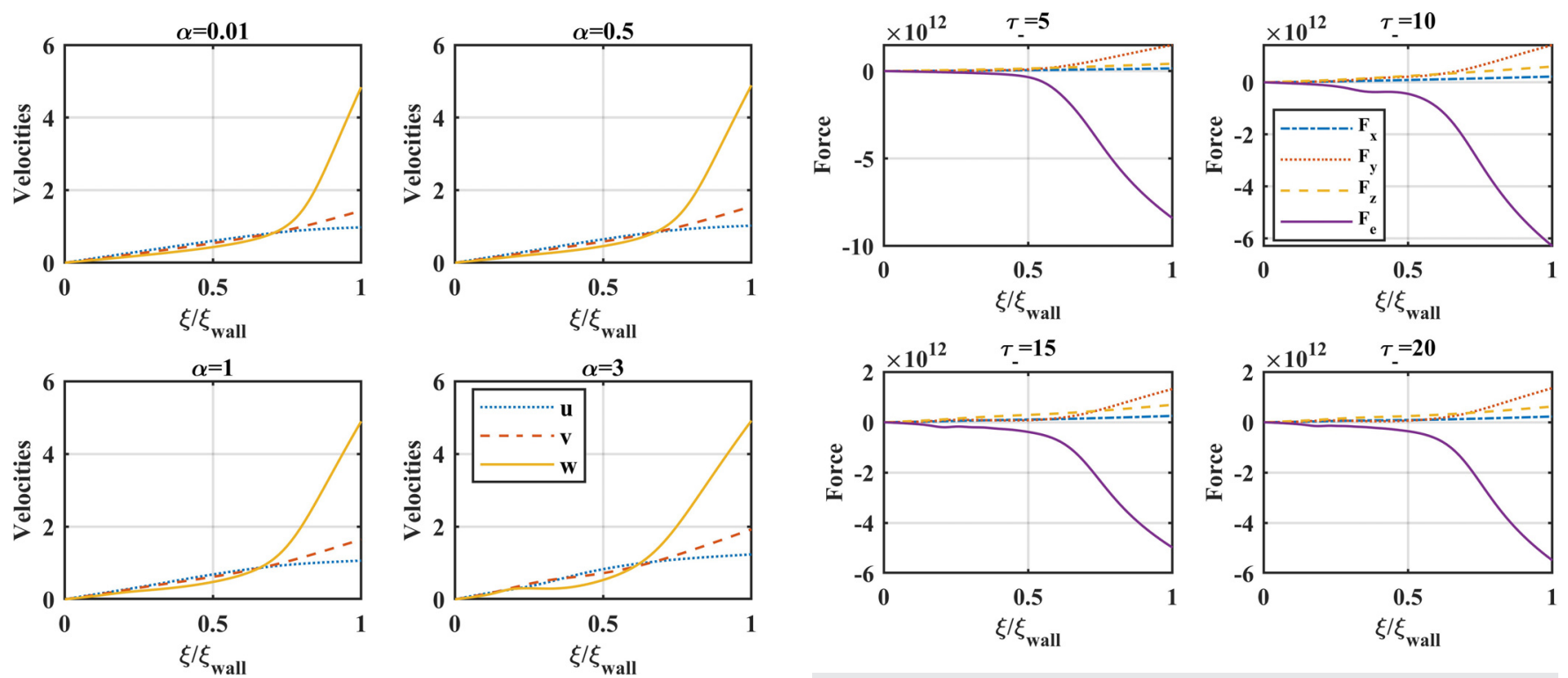

FIG. 11. Normalized positive ion velocity variation along the normalized $z$ axis.
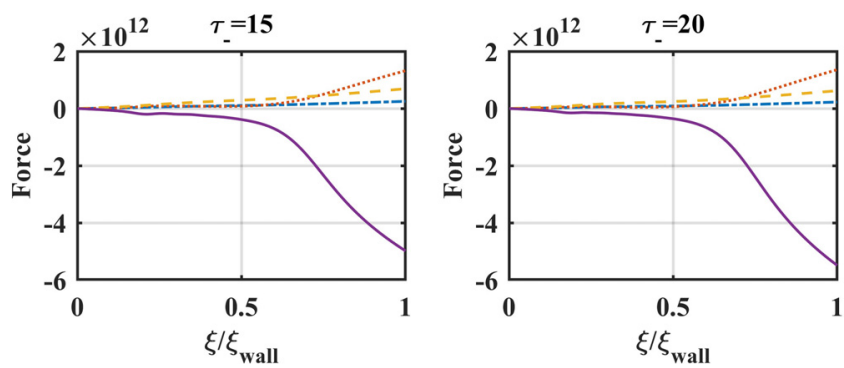

FIG. 13. Normalized Lorentz force variation along the normalized $z$ axis for different $\tau_{-}$for $\alpha=3$. 
force. This enhances the particle density inside the sheath, therefore increasing the sheath width.

\section{CONCLUSIONS}

A numerical study on a sheath using a fluid approach has been carried out for a collisionless magnetized electronegative plasma. The effect of the electronegativity and negative ion temperature on the sheath has been explored. The following conclusions can be drawn from the study:

(a) For a particular negative ion temperature, different regimes of electronegativity have been examined. It is found that for a highly electronegative plasma, the sheath width is maximum.

(b) On increasing the electronegativity, the charge separation has been observed following an increase in the particle density inside the sheath and thereby increasing the thickness.

(c) The dynamics of the positive ion has been affected by the presence of the negative ions.

(d) The magnetic force along the y-direction was seen to increase with the increase in the electronegativity, and on the contrary, the electric force was lowered. With the $\mathbf{E} \times \mathbf{B}$ drift, acting perpendicular to the $x-z$ plane, the $y$-component of velocity has also been increasing monotonically.

(e) For the colder negative ions, it is noted that the sheath widens. Also, they have seen to impact the electric force. The electric force decreases with the decrease in the negative ion temperature.

(f) The present work may have useful applications in processing plasmas as well as in fusion related studies. Additionally, the role of the thermal ions can be incorporated for further study.

\section{ACKNOWLEDGMENTS}

The authors would like to acknowledge the Department of Atomic Energy, Government of India for the financial support for the present work.

\section{DATA AVAILABILITY}

The data that support the findings of this study are available from the corresponding author upon reasonable request.

\section{REFERENCES}

${ }^{1}$ R. Moulick, S. Adhikari, and K. Goswami, Phys. Plasmas 26, 043512 (2019).

${ }^{2}$ M. Khoramabadi, H. Ghomi, and P. K. Shukla, J. Appl. Phys. 109, 073307 (2011).

${ }^{3}$ K. U. Riemann, J. Phys. D 24, 493-518 (1991).

${ }^{4} \mathrm{R}$. Chodura, Phys. Fluids 25, 1628-1633 (1982).

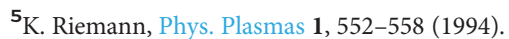

${ }^{6}$ P. C. Stangeby, Nucl. Fusion 52, 083012 (2012).

${ }^{7}$ M. Aslaninejad and K. Yasserian, Phys. Plasmas 19, 033504 (2012).

${ }^{8}$ M. M. Hatami, B. Shokri, and A. R. Niknam, Phys. Plasmas 15, 123501 (2008).

${ }^{9}$ R. L. F. Boyd, J. B. Thompson, and H. S. W. Massey, "The operation of Langmuir probes in electro-negative plasmas," Proc. R. Soc. London, Ser. A 252, 102-119 (1959).

${ }^{10}$ E. Stoffels, W. W. Stoffels, and G. M. W. Kroesen, Plasma Sources Sci. Technol. 10, 311-317 (2001).

${ }^{11}$ J. I. Fernández Palop, J. Ballesteros, V. Colomer, and M. A. Hernández, J. Appl, Phys. 80, 4282-4291 (1996).

${ }^{12}$ J. P. J. Dubois, K. Achkasov, D. Kogut, A. Ahmad, J. M. Layet, A. Simonin, and G. Cartry, J. Appl. Phys. 119, 193301 (2016).

${ }^{13}$ S. S. H. Ohtake, Appl. Phys. Lett. 68, 2416 (1996).

${ }^{14}$ K. Yasserian and M. Aslaninejad, Phys. Plasmas 17, 023501 (2010).

${ }^{15} \mathrm{M}$. Bacal and M. Wada, Appl. Phys. Rev. 2, 021305 (2015).

${ }^{16}$ S. Béchu, A. Soum-Glaude, A. Bés, A. Lacoste, P. Svarnas, S. Aleiferis, A. A. Ivanov, and M. Bacal, Phys. Plasmas 20, 101601 (2013).

${ }^{17}$ B. Kakati, S. S. Kausik, M. Bandyopadhyay, B. K. Saikia, and P. K. Kaw, Sci. Rep. 7, 11078 (2017).

${ }^{18}$ R. Franklin and J. Snell, J. Phys. D 32, 1031 (1999).

${ }^{19}$ N. Oudini, J.-L. Raimbault, P. Chabert, A. Meige, and A. Aanesland, Phys. Plasmas 20, 043501 (2013).

${ }^{20}$ T. E. Sheridan, P. Chabert, and R. W. Boswell, Plasma Sources Sci. Technol. 8, 457 (1999).

${ }^{21}$ T. E. Sheridan, J. Phys. D 32, 1761 (1999).

${ }^{22}$ T. E. Sheridan, N. S. J. Braithwaite, and R. W. Boswell, Phys. Plasmas 6, 4375-4381 (1999).

${ }^{23}$ M. Lampe, W. M. Manheimer, R. F. Fernsler, S. P. Slinker, and G. Joyce, Plasma Sources Sci. Technol. 13, 15 (2004).

${ }^{24}$ R. Moulick and K. S. Goswami, Phys. Plasmas 22, 033510 (2015).

${ }^{25} \mathrm{P}$. D. Edgley, A. von Engel, and M. A. Grace, "Theory of positive columns in electronegative gases,” Proc. R. Soc. London, Ser. A 370, 375-387 (1980).

${ }^{26}$ R. Deutsch and E. Räuchle, Phys. Rev. A 46, 3442-3453 (1992).

${ }^{27}$ K. Yasserian, M. Aslaninejad, M. Ghoranneviss, and F. M. Aghamir, J. Phys. D 41, 105215 (2008).

${ }^{28}$ K. Yasserian, M. Aslaninejad, and M. Ghoranneviss, Phys. Plasmas 16, 023504 (2009).

${ }^{29}$ R. Moulick, S. Adhikari, and K. S. Goswami, Phys. Plasmas 24, 114501 (2017).

${ }^{30}$ H.-B. Valentini, Plasma Sources Sci. Technol. 9, 574 (2000).

${ }^{31}$ J. R. Forrest and R. N. Franklin, J. Phys. D 1, 1357 (1968).

${ }^{32}$ B. Remya, R. V. Reddy, B. T. Tsurutani, G. S. Lakhina, and E. Echer, J. Geophys. Res.: Space Phys. 118, 785-793, https://doi.org/10.1002/jgra.50091 (2013).

${ }^{33}$ V. V. Mikhailenko, V. S. Mikhailenko, and H. J. Lee, Phys. Plasmas 23, 112122 (2016).

${ }^{34}$ I. I. Beilis and M. Keidar, Phys. Plasmas 5, 1545-1553 (1998).

${ }^{35}$ R. N. Franklin, J. Plasma Phys. 78, 21 (2012).

${ }^{36}$ F. F. Chen, "Comment on 'Comment on "Magnetic field effects on gas discharge plasmas" [Phys. Plasmas 14, 024701 (2007)],"” Phys. Plasmas 14, 094703 (2007).

${ }^{37}$ R. M. Crespo and R. N. Franklin, Plasma Sources Sci. Technol. 23, 035012 (2014).

${ }^{38}$ J. Jing-Ju Li and Z. An Wei, Phys. Plasmas 20, 063503 (2013). 